\title{
Orientation-Aware Indoor Localization using Affinity Propagation and Compressive Sensing
}

\author{
Chen Feng ${ }^{1,2}$, Wain Sy Anthea $\mathrm{Au}^{1}$, Shahrokh Valaee ${ }^{1}$, and Zhenhui Tan ${ }^{2}$ \\ ${ }^{1}$ Department of Electrical and Computer Engineering, University of Toronto \\ ${ }^{2}$ State Key Laboratory of Rail Traffic Control and Safety, Beijing Jiaotong University \\ Email: \{chenfeng, anthea, valaee\}@comm.utoronto.ca, zhhtan@center.njtu.edu.cn
}

\begin{abstract}
The sparse nature of location finding makes it desirable to exploit the theory of compressive sensing for indoor localization. In this paper, we propose a received signal strength (RSS)-based localization scheme in Wireless Local Area Networks (WLANs) using the theory of compressive sensing (CS), which offers accurate recovery of sparse signals from a small number of measurements by solving an $\ell_{1}$-minimization problem. In order to mitigate the effects of RSS variations due to channel impediments and mobile device orientation, a two-step localization scheme is proposed by exploiting affinity propagation for coarse localization followed by a CS-based fine localization to further improve the accuracy. We implement the localization algorithm on a WiFi-integrated mobile device to evaluate the performance. Experimental results indicate that the proposed system leads to substantial improvements on localization accuracy and complexity over the widely used traditional fingerprinting methods.
\end{abstract}

Keywords- Indoor localization, Compressive sensing, Affinity propagation, WLANs

\section{INTRODUCTION}

Accurate indoor localization for mobile users is one of the fundamental and challenging problems in signal processing [1]. Among the localization algorithms, Received Signal Strength (RSS)-based approaches have been extensively studied as an inexpensive solution for delivering indoor Location-Based Services (LBSs). Fingerprinting is frequently used by WLAN positioning techniques to generate a location-dependent database of the environment [2]. The position of the mobile user is then estimated by comparing its online readings with the database. However, since the indoor propagation channel varies over time [3], online RSS measurements may deviate from those stored in the database.

One simple solution to compensate for the radio channel impediments is the k-nearest neighbor algorithm $(\mathrm{kNN})$, which estimates the mobile user's location by computing the centroid of the $\mathrm{k}$ closest neighbors which have the smallest Euclidean distance with respect

This work was supported by the State Key Laboratory of Rail Traffic Control and Safety, Beijing Jiaotong University, under project No.863 (2007AA01Z277). to the online RSS readings [4]. Such system is easy to implement but the estimation is not very accurate. Another effective approach is to statistically analyze the probabilities of each potential position using the Bayesian theory [5] or kernel functions [6]. However, an explicit formulation of RSS distribution is challenging in real environments and thus, these probabilistic-based systems often have a high computational complexity.

Furthermore, the orientation of the mobile device may significantly change the RSS measurement even at one particular position. Unfortunately, the literature on orientation-aware methodologies without using a specialized hardware like a digital compass is only limited to either getting an integrated database by averaging RSS observations across different orientations [7], or predicting a propagation model by taking care of the attenuation factors for different orientations [8].

Compressive Sensing (CS) provides a novel framework for recovering sparse or compressible signals, with far fewer noisy measurements than that needed by the Nyquist sampling theorem [9][10]. The sparse signal can be reconstructed exactly with high probability by solving an $\ell_{1}$-minimization problem [11]. The sparse nature of location finding in the spatial domain motivates us to exploit the theory of CS for indoor localization.

In this paper, we propose a RSS-based localization scheme composed of two phases: an offline phase, during which clustering by affinity propagation is performed to address the effects of RSS variation and mobile device orientation; and an online phase, which consists of a coarse localization stage using cluster matching and a fine localization stage using compressive sensing. We have implemented the proposed localization scheme on a Personal Digital Assistant (PDA, HP iPAQ hx4700 with Windows Mobile 2003 pocket PC) to evaluate the localization accuracy. Experimental results indicate that the proposed scheme outperforms the kNN and Kernelbased localization algorithms [4][6].

The remainder of this paper is organized as follows. Section II sets up the problem, and describes the overview of the proposed localization scheme. Sec- 
tion III and Section IV present the offline phase by affinity propagation, and the online phase by cluster matching and compressive sensing, respectively. The performance is evaluated through implementations in Section V. Finally, Section VI concludes the paper.

\section{Problem Setup}

Consider a scenario, where a mobile device equipped with a WLAN adapter takes RSS measurements from $L$ WLAN Access Points (APs) with unknown positions. In a fingerprinting-based localization system, time samples of RSS readings from different APs are collected at known locations, which are referred to as the Reference Points (RPs), and such a database is referred to as the radio map. In practical application, the radio map can be stored in a location server and transmitted along with the layout of the environment to the mobile device when the terminal enters the coverage area of the server. For the brevity of discussion in this paper, we ignore the interactions with the server and simply assume that the radio map and the layout of the environment is available at the mobile device.

Since we consider the dependancy of the signal strength on orientations, in our technique the RSS measurements are collected at each RP by pointing the mobile device to four different orientations (i.e., north, south, east, west). Such a radio map gives a sufficient representation of the spatial RSS properties in the given environment. The radio map database is denoted by the $L \times N$ matrix $\Psi^{(o)}$ :

$$
\Psi^{(o)}=\left(\begin{array}{cccc}
\psi_{1,1}^{(o)} & \psi_{1,2}^{(o)} & \cdots & \psi_{1, N}^{(o)} \\
\psi_{2,1}^{(o)} & \psi_{2,2}^{(o)} & \cdots & \psi_{2, N}^{(o)} \\
\vdots & \vdots & \ddots & \vdots \\
\psi_{L, 1}^{(o)} & \psi_{L, 2}^{(o)} & \cdots & \psi_{L, N}^{(o)}
\end{array}\right)
$$

where $\psi_{i j}^{(o)}$ represents an average of RSS readings over time domain from AP $i$ at $\mathrm{RP} j$ at a specific orientation $o$, where $i=1,2, \ldots, L, j=1,2, \ldots, N$, and $o \in \mathcal{O}=$ $\left\{0^{\circ}, 90^{\circ}, 180^{\circ}, 270^{\circ}\right\} . L$ is the total number of APs and $N$ is the total number of RPs. The columns of $\Psi^{(o)}$ can be referred to as $\psi_{j}^{(o)}, j=1,2, \ldots, N$, which represent the RSS readings at each RP $j$.

We propose a two-phase localization scheme: an offline phase in which RSS readings at different reference points are collected and clustered using affinity propagation, and an online phase consisting of coarse and fine localization steps. Coarse localization mitigates the effects of RSS variations due to channel impediments and device orientation, and removes potential outliers by confining the localization problem into a region, in which only the nearby RPs with the most possible orientations are included. A CS-based fine localization scheme is then used for accurate location estimation. The overview of the proposed localization scheme is shown in Fig. 1. In the following sections, we discuss each block in details.

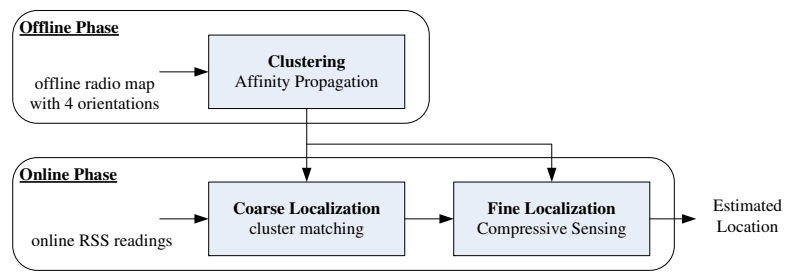

Fig. 1. The overview of the proposed localization scheme.

\section{Offline Phase: Clustering By AfFinity PROPAGATION}

Affinity propagation [12] is an algorithm that, taking into account an input measure of similarity between pairs of data points, recursively transmits real-valued messages between the neighboring data points until a set of exemplars and corresponding clusters emerges. There are two kinds of real-valued messages transmitted: the responsibility message and the availability message. Because of the space limitation, we do not review the affinity propagation algorithm here and refer the interested readers to [12]. Central to the affinity propagation technique is the similarity function, which we define to be the negative summation of squared RSS differences between two RPs,

$$
\begin{array}{r}
s(i, j)^{(o)}=-\sum_{\ell=1}^{L}\left\|\psi_{\ell i}^{(o)}-\psi_{\ell j}^{(o)}\right\|^{2}, \\
\forall i, j \in\{1,2, \ldots, N\}, \quad o \in \mathcal{O} .
\end{array}
$$

In order to consider the dependence of signal strength on orientations, we apply the affinity propagation algorithm on the four radio maps with different orientations separately, to generate clusters and their corresponding exemplars. For each orientation $o$, let us represent by $\mathcal{C}_{j}^{(o)}$ the set of cluster members with RP $j$ as their exemplar, and by $\mathcal{H}^{(o)}$ the set of all exemplars. The clustering results are tabulated for the use in the localization step.

\section{Online Phase: Localization}

The online phase consists of two stages: a coarse localization stage using cluster matching, and a fine localization stage using compressive sensing.

\section{A. Coarse Localization Stage: Cluster Matching}

The mobile device reads the RSS information of all exemplars across four orientations, and computes the 
similarity between its online RSS observations and each exemplar to find the best cluster, denoted as $\mathcal{C}$,

$$
\mathcal{C}=\mathcal{C}_{\hat{j}}^{(\hat{o})}, \text { where }\{\hat{j}, \hat{o}\}=\arg \max _{o \in \mathcal{O}} \max _{j \in \mathcal{H}^{(o)}} s\left(r, \psi_{j}^{(o)}\right)
$$

where $r$ is the online RSS reading. The RPs in $\mathcal{C}$ are then used for fine localization as will be discussed in the next subsection. Note that if the radio map is stored in the server, the two step localization scheme reduces the communication cost since only the RSS readings of the set of exemplars and the selected cluster $\mathcal{C}$ are needed to be transmitted to the mobile node.

\section{B. Fine Localization Stage: Compressive Sensing}

For the moment, we assume that the mobile user is located at one of the selected RPs. We will relax this assumption shortly. Let $|\mathcal{C}|=N^{\prime}$. The user's location can be formulated as a 1 -sparse $N^{\prime} \times 1$ vector $\theta$, with all elements equal to zero except $\theta(n)=1$, where $n$ is the index of the RP at which the mobile user is located, namely,

$$
\theta=[0, \ldots, 0,1,0, \ldots, 0]^{T}
$$

where the superscript $T$ denotes transposition. We further define a matrix $\Phi$ as the AP selection operator. Each row of $\Phi$ is a $1 \times L$ vector with all elements equal to zero except $\phi(\ell)=1$, where $\ell$ is the index of the AP selected for positioning,

$$
\Phi_{m}=[0, \ldots, 0,1,0, \ldots, 0], \forall m=1,2, \ldots, M
$$

where $M$ is the number of APs used for positioning. In our implementation, for simplicity, the set of APs with the strongest online RSS readings is selected.

Based on the definitions above, the online RSS reading measured by the mobile device can be expressed as:

$$
y=\Phi \Psi \theta+\varepsilon
$$

where $\varepsilon$ is the measurement noise, and $\Psi$ is obtained from the database following the cluster matching by coarse localization.

Besides sparsity, incoherence is another principle that is required by the CS theory for accurate signal recovery [9]. However, in our case, $\Psi$ and $\Phi$ are coherent in the spatial domain. In order to induce the incoherence needed by the CS theory, we propose to use the preprocessor $T$ (i.e., $Y=T y$ ), which is defined as:

$$
T=Q R^{\dagger}
$$

where $R=\Phi \Psi$, and $Q=\operatorname{orth}\left(R^{T}\right)^{T}$, where $\operatorname{orth}(R)$ is an orthogonal basis for the range of $R$, and $R^{\dagger}$ is the pseudo-inverse of matrix $R$. We proved in our previous work [13][14] that if $M$ is in the order of $\log \left(N^{\prime}\right)$, the minimum bound required by the $\mathrm{CS}$ theory [9], $\theta$ can be well recovered from $Y$ with very high probability, ${ }^{3}$ through the following $\ell_{1}$-minimization program.

$$
\hat{\theta}=\arg \min _{\theta \in R^{N^{\prime}}}\|\theta\|_{1} \text {, s.t. } Y=Q \theta+\varepsilon^{\prime} .
$$

where $\varepsilon^{\prime}=T \varepsilon$. That is, if the mobile user is located at one of the RPs (1-sparse nature), the recovered position is almost exact.

In practice, however, the mobile user may not necessarily be located at an RP. In such cases, the recovered location $\hat{\theta}$ is not a 1 -sparse vector, rather a vector with a few non-zero coefficients. In order to compensate for the error induced by the grid assumption, a post-processing procedure is conducted. We choose the dominant coefficients in $\hat{\theta}$ whose values are above a certain threshold $\lambda$, and take the centroid of these RPs as the estimated location.

\section{EXPERIMENTAL RESUlTS}

This section provides details on the experimental evaluation of the proposed method using a real radio map database obtained from an office building that reflects a typical setting of indoor environment. Specifically, the experiments were carried out on a $12 \mathrm{~m} \times 36 \mathrm{~m}$ area of the fourth floor of an eight-story building (Bahen Center at the University of Toronto). A total of 17 APs were detected in the measurement area. A PDA (HP iPAQ hx4700 with Windows Mobile 2003 Pocket PC) was used to measure the WLAN signal strength value, and a software was developed in Visual Studio C\# to implement the localization scheme on the device [15]. The RSS observations from 17 APs were recorded for a period of 100 seconds (one reception per second) over 50 RPs across four different orientations at each RP during the offline phase.

We studied the benefits of orientation-aware affinity propagation and compressive sensing on the performance of positioning, in terms of the localization error. The localization error is measured by averaging the Euclidean distance between the estimated location of the mobile user and its true location.

Fig. 2 illustrates the localization error of our orientation-aware localization scheme averaged over 50 independent runs, with respect to the number of APs. The implementation result shows that our proposed system, which performs clustering on the radio maps with four orientations separately, always achieves better performance than a similar algorithm operating on an integrated radio map, obtained by averaging RSS readings across different orientations.

As mentioned in Section IV-A, the affinity propagation allows the reduction in the number of APs used for location estimation. Since affinity propagation reduces the area of interest into a subset $\mathcal{C}$, the dimension of our 


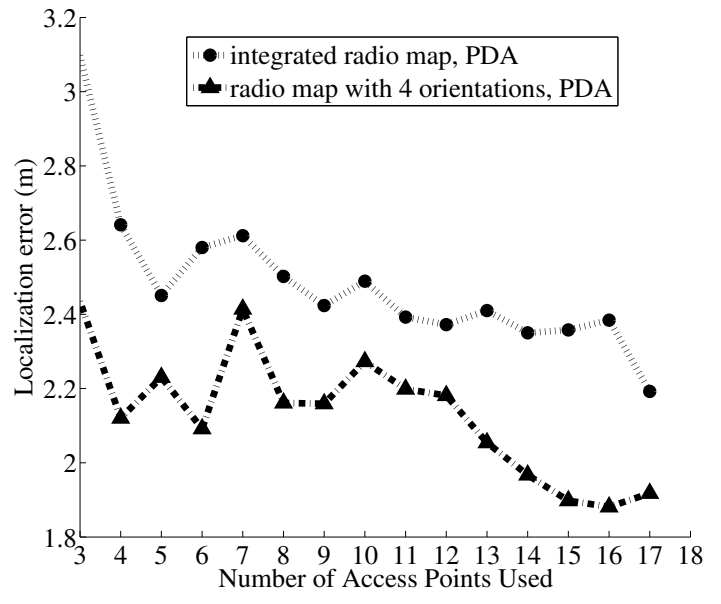

Fig. 2. The localization error, with respect to the number of access points used in two algorithms.

sparse signal is also reduced to $N^{\prime}$. This allows the system to reduce the number of APs, required for accurate location recovery. As illustrated in Fig. 2, only 4 APs are needed to achieve $2.1 \mathrm{~m}$ error, when clusters with about 15 members are generated by affinity propagation in our approach. We note that 4 APs $(M=4)$ is approximately the minimum bound of $\log \left(N^{\prime}\right) \approx 3.9$ as required by the CS theory.

We also compare the performance of the proposed localization scheme with the $\mathrm{kNN}(\mathrm{k}=3)$ and the Kernelbased fingerprinting methods [6]. Fig. 3 shows the implementation result on the PDA. It is shown that when 4 APs are used, the proposed two-phase localization scheme leads to $46 \%(1.8 \mathrm{~m})$ and $28 \%(0.8 \mathrm{~m})$ improvements over the kNN and Kernel-based algorithms, respectively.

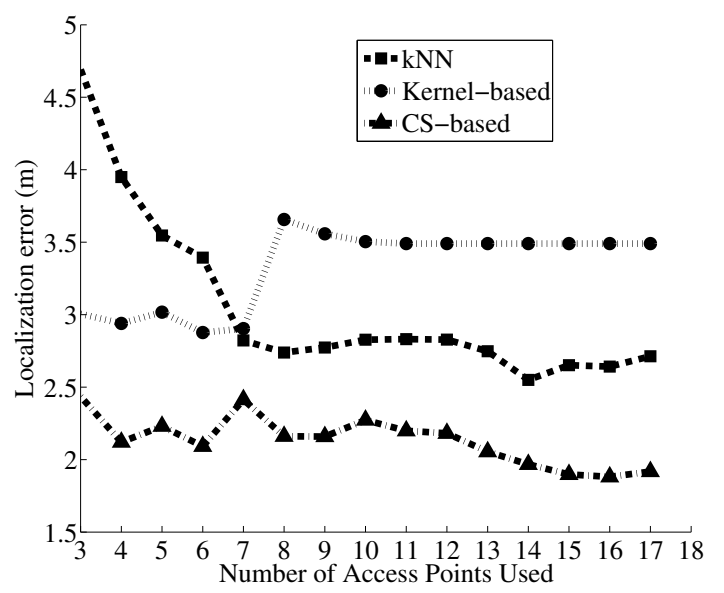

Fig. 3. Comparison of localization error evaluated on PDA.

\section{CONCLUSION}

In this paper, we have proposed an orientationaware indoor localization scheme using affinity propagation and compressive sensing in WLANs. The intuition behind this technique is that localization is a sparse problem and thus according to the CS theory, the location can be well estimated from only a small number of noisy measurements through an $\ell_{1}$-minimization program. We have used a coarse localization by exploiting affinity propagation to compensate for the effects of complex indoor radio channel and the orientation of the mobile device, and a pre-processing procedure to ensure incoherence needed in the CS theory during the fine localization stage. Experimental results demonstrate that the proposed CS method achieves a high level of localization accuracy.

\section{REFERENCES}

[1] N. Patwari, J. N. Ash, and S. Kyperountas, "Locating the nodes: Cooperative localization in wireless sensor networks," IEEE Signal Processing Magazine, pp. 54-69, July 2005.

[2] K. Kaemarungsi and P. Krishnamurthy, "Modeling of indoor positioning systems based on location fingerprinting," Proc. INFOCOM, vol. 2, pp. 1012-1022, 2004.

[3] A. Goldsmith, Wireless Communications, 1st ed. Cambridge University Press, 2005.

[4] B. Li, J. Salter, A. G. Dempster, and C. Rizos, "Indoor positioning techniques based on wireless lan," Auswireless Conference, 2006.

[5] R. Singh, L. Macchi, C. Regazzonik, and K. Plataniotis, "A statistical modelling based location determination method using fusion in wlan," Proc. Int'l Workshop Wireless Ad-Hoc Networks, 2005.

[6] A. Kushki, N. Plataniotis, and A. N. Venetsanopoulos, "Kernelbased positioning in wireless local area networks," IEEE Trans. on Mobile Computing, vol. 6, no. 6, June 2007.

[7] P. Bahl and V. Padmanabhan, "Radar: An in-building rf-based user location and tracking system," Proc. INFOCOM, vol. 2, pp. 775-784, 2002.

[8] M. L. Han, T. Tsuchiya, and K. Koyanagi, "Orientation-aware indoor localization path loss prediction model for wireless sensor networks," NBiS, pp. 169-178, 2008.

[9] J. C. Emmanuel and B. W. Michael, "An introduction to compressive sampling," IEEE Signal Processing Magazine, pp. 21-30, March 2008.

[10] J. Romberg, "Imaging via compressive sampling," IEEE Signal Processing Magazine, pp. 14-20, March 2008.

[11] S. S. Chen, D. L. Donoho, and M. A. Saunders, "Atomic decomposition by basis pursuit," SIAM Journal on Scientific Computing, vol. 20, no. 1, pp. 33-61, 1998.

[12] B. J. Frey and D. Dueck, "Clustering by passing messages between data points," Science, vol. 315, no. 1, pp. 972-976, February 2007.

[13] C. Feng, S. Valaee, and Z. H. Tan, "Multiple target localization using compressive sensing," IEEE Globecom, 2009.

[14] _ "Localization of wireless sensors using compressive sensing for manifold learning," IEEE The 20th Personal, Indoor and Mobile Radio Communications Symposium, PIMRC, 2009.

[15] "Opennetcf shared source projects," 2009. [Online]. Available: http://www.opennetcf.com/FreeSoftware/tabid/84/Default.aspx 